PACIFIC JOURNAL OF MATHEMATICS

Vol. 181, No. 3, 1997

\title{
SOME PROPERTIES OF CHARACTER SHEAVES
}

\author{
Anne-Marie Aubert
}

\author{
Dedicated to the memory of Olga Taussky-Todd
}

\section{Introduction.}

In 1986, George Lusztig stated some interesting properties of character sheaves with non-zero restriction to the unipotent variety and proved them for the group $S O_{2 n+1}\left(\mathbb{F}_{q}\right)$ under the assumption that the characteristic $p$ of $\mathbb{F}_{q}$ is good. In February 1992, the author has given lectures on that proof of Lusztig in the Seminar "Faisceaux Caractères" at the École Normale Supérieure and it was no doubt for her that the same method should work for the other types of groups (both classical and exceptional), as it has been already noticed by Lusztig. It is one purpose of this paper to show that the question can be reduced to statements uniquely involving representations of some Coxeter groups. In particular we shall show that the method employed by Lusztig for the group $S O_{2 n+1}\left(\mathbb{F}_{q}\right)$ provides a general process (based on both "parabolic induction" of character sheaves and generalized Springer correspondence) for associating a well-defined unipotent class to any character sheaf which has non-zero restriction to the unipotent variety.

A different map from the (all) set of character sheaves to the set of unipotent classes has been defined by Lusztig in 1992, under the assumption that $p$ is "large enough" (that is sufficiently large, so that we can operate with the Lie algebra of $G$ as if we were in characteristic zero). That map is the composition of two maps: One from character sheaves to a set of parameters in the Langlands dual of $G$ and one from this set of parameters to unipotent classes in $G$. The images of a given character sheaf by these two maps coincide (that result gives an answer to a question that J. Arthur asked the author in June 1995: It is a first step in the understanding of how the two classifications of character sheaves given by Lusztig correspond together).

It is a pleasure to thank Gunter Malle and Jean-Loup Waldspurger who carefully read an earlier version of this paper and suggested improvements. 


\section{Preliminaries on character sheaves.}

Let $k$ be an algebraic closure of a finite field $\mathbb{F}_{q}$ of $q$ elements. We fix a prime number $\ell$ invertible in $k$. Let $G$ be a connected reductive algebraic group over $k$. We assume that the characteristic $p$ of $k$ is good for $G$. Let $T$ be a maximal torus of $G$ and $W:=\mathrm{N}_{G}(T) / T$ the Weyl group $G$ with respect to $T$.

Following Lusztig [7], we shall review a definition of character sheaves on $G$. Let $\mathcal{D} G$ be the bounded derived category of constructible $\overline{\mathbb{Q}}_{\ell}$-sheaves on $G$, and let $\mathcal{M} G$ be the full subcategory of $\mathcal{D} G$ consisting of perverse sheaves. Let $\mathcal{S}(T)$ be the set of isomorphisms classes of local systems $\mathcal{L}$ on $T$ such that rank $\mathcal{L}=1$ and such that $\mathcal{L}^{\otimes e} \simeq \overline{\mathbb{Q}}_{\ell}$ for some integer $e \geq 1$, invertible in $k$. Take a local system $\mathcal{L}$ in $\mathcal{S}(T)$ such that $w^{*} \mathcal{L} \simeq \mathcal{L}$ for some $w \in W$. Then one can construct a complex $K_{w}^{\mathcal{L}} \in \mathcal{D} G$ as in [7, 12.1]. For each $\mathcal{L} \in \mathcal{S}(T)$, we denote by $\hat{G}_{\mathcal{L}}$ the set of isomorphism classes of irreducible perverse sheaves $A$ on $G$ such that $A$ is a constituent of the $i$-th cohomology perverse sheaf ${ }^{p} H^{i}\left(K_{w}^{\mathcal{L}}\right)$ of $K_{w}^{\mathcal{L}}$ for some $w$ and some $i \in \mathbb{Z}$. The set $\hat{G}$ of character sheaves of $G$ is defined as the union of $\hat{G}_{\mathcal{L}}$, where $\mathcal{L}$ runs over all the elements in $\mathcal{S}(T)$.

Let $A$ be a character sheaf in $\hat{G}_{\mathcal{L}}$. Assume chosen, once and for all, an isomorphism of abstract groups from the set of isomorphisms classes of tame local systems of rank one on $k^{\times}$onto $k^{\times}$, this gives rise to an isomorphism $\mathcal{S}(T) \stackrel{\sim}{\rightarrow} T^{*}$ where $T^{*}$ is the dual group of $T$ (see [10, Section 1.6]), hence the local system $\mathcal{L}$ which is associated by Lusztig with the character sheaf $A$ can be interpreted as a semisimple element $s$ of $G^{*}$, the Langlands dual of $G$.

By [7, Corollary 11.4], there is a well-defined map

$$
\hat{G} \longrightarrow\left\{W \text {-orbits on } T^{*}\right\} .
$$

If $A \in \hat{G}$ corresponds to $s \in T^{*}$ we say that $A$ lies in the series defined by $s$; the subset of $\hat{G}$ consisting of all character sheaves in the series defined by $s$ will be denoted by $\hat{G}_{s}$.

(2.1) Let $L$ be a Levi subgroup of a parabolic subgroup $P$ of $G$ and let $\hat{L}$ be the set of character sheaves on $L$. In [7, 4.1], Lusztig introduced the notion of induction $\operatorname{ind}_{L}^{G}$ of character sheaves. In particular, for each $A \in \hat{L}_{s}, \operatorname{ind}_{L}^{G} A$ is a semisimple perverse sheaf on $G$, and each irreducible direct summand is a character sheaf which belongs to $\hat{G}_{s}$ (see [7, Proposition 4.8 (b)]).

(2.2) A character sheaf on $G$ is said to be cuspidal if it is not contained in $\operatorname{ind}_{L}^{G} A$ for any Levi subgroup $L$ of a proper parabolic subgroup of $G$ and any $A \in \hat{L}$. Since $p$ is good (almost good would be sufficient here), it is known by 
[7] that, for any cuspidal pair $(\Sigma, \mathcal{E})$, the (shift of) intersection cohomology complex $\operatorname{IC}(\bar{\Sigma}, \mathcal{E})[\operatorname{dim} \Sigma]$, extended to the whole of $G$ by zero on $G-\bar{\Sigma}$, is a cuspidal character sheaf on $G$. All the cuspidal character sheaves on $G$ are obtained in this way.

Let $G_{\text {uni }}$ be the unipotent variety of $G$. Let $\hat{G}^{0}$ be the set of cuspidal character sheaves on $G$. Let $W_{s}$ be the Weyl group of $C_{G^{*}}(s)$ (with respect to $T^{*}$ ), identified with a subgroup of $W$.

Fact 2.3. Assume that $G$ is simple of adjoint type (with $\operatorname{rank} G \neq 0$ ). Then $\hat{G}_{s}$ contains at most one cuspidal character sheaf with non-zero restriction to $G_{\text {uni }}$ and such a character sheaf exists exactly in the following cases:

\begin{tabular}{|c|c|c|}
\hline Type of $G$ & Condition on $n$ & Type of $W_{s}$ \\
\hline$B_{n}$ & $n=2 t(t+1)$ & $C_{t(t+1)} \times C_{t(t+1)}$ \\
$C_{n}$ & $n=2 t(4 t \pm 1)$ & $D_{4 t^{2}} \times B_{4 t^{2} \pm 2 t}$ \\
$D_{n}$ & $n=8 t^{2}$ & $D_{4 t^{2}} \times D_{4 t^{2}}$ \\
$G_{2}$ & & $G_{2}$ \\
$F_{4}$ & & $F_{4}$ \\
$E_{8}$ & & $E_{8}$ \\
\hline
\end{tabular}

where $t \geq 1$.

Proof. It can be trivially extracted (see the appendix) from the explicit classification of cuspidal characters sheaves provided by Lusztig in [7], Section 23 .

Remark 2.4. Fact 2.3 has been already noticed by Lusztig in [8], (4.1) and (7.11).

(2.5) Any character sheaf of $G$ is obtained as a direct summand of $\operatorname{ind}_{L}^{G} A$ for the Levi complement $L$ of some parabolic subgroup $P$ of $G$ and a cuspidal character sheaf $A$ on $L$.

Let $L$ be a Levi subgroup of a parabolic subgroup of $G$ such that $\hat{L}^{0}$ is non-emtpy. We set

$$
W_{L}^{G}:=\mathrm{N}_{G}(L) / L
$$

Then, by [6, Theorem 9.2 (a)], the group $W_{L}^{G}$ is a finite Coxeter group. Now let $s \in T^{*}$ such that $\hat{L}_{s}^{0}$ is not empty, and let $A \in \hat{L}_{s}^{0}$.

By $[14,(5.16 .1)$ and II, proof of (4.21)] (see also [5, (8.5)]), the stabilizer of $A$ in $W_{L}^{G}$ is the image of the canonical map

$$
\left(\mathrm{N}_{G^{*}}\left(L^{*}\right) \cap \mathrm{C}_{G^{*}}(s)\right) / \mathrm{C}_{L^{*}}(s) \hookrightarrow \mathrm{N}_{G^{*}}\left(L^{*}\right) / L^{*},
$$


where $L^{*} \subseteq G^{*}$ denotes the standard Levi subgroup dual to $L$ and $\mathrm{C}_{G^{*}}(s)$ (resp. $\mathrm{C}_{L^{*}}(s)$ ) denotes the centralizer of $s$ in $G^{*}$ (resp. $L^{*}$ ). We see that this stabilizer only depends on $G, s$ and $L$, and we shall therefore denote it by $W_{L, s}^{G}$.

(2.6) Let $L$ be a Levi subgroup of some parabolic of $G$ and let $s \in T^{*}$ be a semisimple element. Let $\hat{L}_{\text {uni }}^{0}$ be the subset of all $A \in \hat{L}^{0}$ such that $A$ has non-zero restriction to $L_{\text {uni }}$.

Assume that $s$ isolated in $G^{*}$ (i.e., such that $\mathrm{C}_{G^{*}}(s)$ has the same semisimple rank as $\left.G^{*}\right)$ and that the set $\hat{L}_{s}^{0} \cap \hat{L}_{\text {uni }}^{0}$ is non-empty.

Then, if $G$ is of exceptional type, Fact 2.3 shows us that $L=T$ (which implies that $W_{L}^{G}=W$ and $W_{L, s}^{G}=W_{s}$ ), if $G$ is of classical type the descriptions of the groups $W_{L}^{G}$ (see [6], (12.1.3) and (13.1.3)) and $W_{L, s}^{G}$ (see [8], Section 4.1) are as follows.

\begin{tabular}{|c|c|c|}
\hline Type of $G$ & Type of $W_{L}^{G}$ & Type of $W_{L, s}^{G}$ \\
\hline$B_{n}$ & $C_{n-2 t(t+1)}$ & $C_{a} \times C_{b}$ where $a+b=n-2 t(t+1)$ \\
\hline$C_{n}$ & $B_{n-2 t(4 t \pm 1)}$ & $\begin{cases}B_{a} \times B_{b} \text { where } a+b=n-2 t(4 t \pm 1), & \text { if } t \geq 1 \\
D_{a} \times B_{b} \text { where } a+b=n, & \text { if } t=0\end{cases}$ \\
\hline$D_{n}$ & $D_{n-8 t^{2}}$ & $D_{a} \times D_{b}$ where $a+b=n-8 t^{2}$ \\
\hline
\end{tabular}

(2.7) Let $K:=\operatorname{ind}_{L}^{G} A$. Now let $\mathcal{A}:=\operatorname{End}_{\mathcal{M} G} K$ be the endomorphism algebra of $K$ in $\mathcal{M} G$. It is known by [8],(2.4) (a), (2.5) (b), (see also [6, (3.4)]), that $\mathcal{A}$ is isomorphic to the group algebra $\overline{\mathbb{Q}}_{\ell}\left[W_{L, s}^{G}\right]$. Hence we have a decomposition

$$
\operatorname{ind}_{L}^{G}(A)=\sum_{E \in \operatorname{Irr}\left(W_{L, s}^{G}\right)} E \otimes K_{E}^{s}
$$

where $K_{E}^{s} \in \hat{G}_{s}$.

(2.8) Let $\hat{G}_{\text {uni }}$ be the set of isomorphism classes of characters sheaves $A \in \hat{G}$ such that the restriction of $A$ to $G_{\text {uni }}$ is non-zero. Then (2.7) allows us to 
define a bijective map

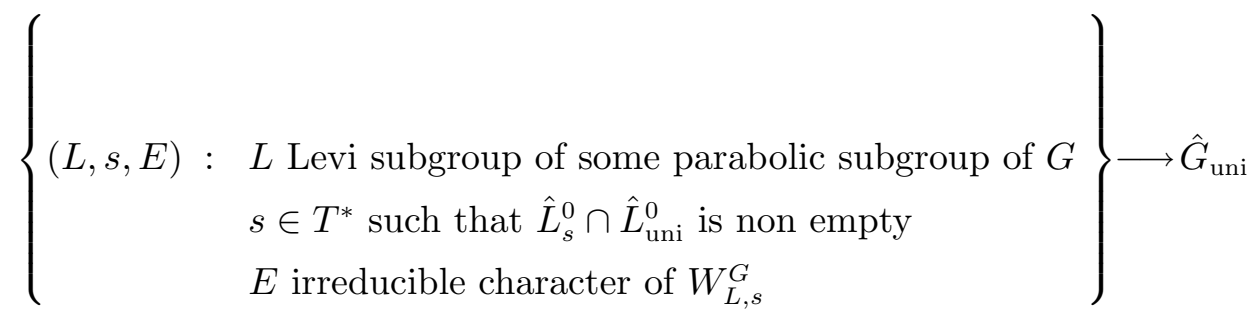

by sending $(L, s, E)$ to $K_{E}^{s} \in \hat{G}_{\text {uni }}$.

(2.9) Let $L_{\text {ad }}$ be the adjoint group of $L$ and let $\pi: L \rightarrow L_{\text {ad }}$ be the canonical map. Let $L_{\text {der }}$ be the derived subgroup of $L$. By [7], (17.10), we can write any $A \in \hat{L}^{0}$ in the form $A=\pi^{*}(\bar{A}) \otimes \mathcal{L}$ where $\bar{A} \in \hat{L}_{\text {ad }}^{0}$ and $\mathcal{L}$ is a tame local system on $L$ which is the inverse image of a local system on $L / L_{\text {der }}$ under the canonical map $L \rightarrow L / L_{\text {der }}$.

We have a corresponding embedding of dual groups $L_{\text {der }}^{*} \subseteq L^{*}$. If $\bar{A}$ lies in the series of $L_{\text {ad }}$ defined by $\bar{s} \in T^{*} \cap L_{\text {der }}^{*}$ and $\mathcal{L}$ corresponds to the central element $z$ of $L^{*}$, then $A$ lies in the series of $L$ defined by $s:=\bar{s} z$. Clearly, we have $\mathrm{C}_{L^{*}}(s)=\mathrm{C}_{L^{*}}(\bar{s})$. Note that if $L=T$ then $\bar{s}=1$.

Assume that $L_{\text {ad }}$ has a cuspidal character sheaf $\bar{A}$ such that $\bar{A}$ has non-zero restriction to the unipotent variety of $L_{\mathrm{ad}}$. Then $\bar{A}$ is uniquely determined, see (2.3). Let $\bar{s} \in T^{*} \cap L_{\text {der }}^{*} \subseteq L^{*}$ such that $\bar{A}$ lies in the series defined by $\bar{s}$. By [6], Theorem 9.2 (b), we have $W_{L, \bar{s}}^{G}=W_{L}^{G}$. We consider the decomposition

$$
\operatorname{ind}_{L}^{G}\left(\pi^{*}(\bar{A})\right)=\sum_{E \in \operatorname{Irr}\left(W_{L}^{G}\right)} E \otimes K_{E}^{\bar{s}}
$$

where $K_{E}^{\bar{s}} \in \hat{G}_{\bar{s}}$ (cf. 2.7).

Let $A \in \hat{L}$ be any cuspidal character sheaf. If the restriction of $A$ to $L_{\text {uni }}$ is zero then the restrictions of all components of $\operatorname{ind}_{L}^{G}(A)$ to $G_{\text {uni }}$ will also be zero (see [8], (2.9)). Assume now that the restriction of $A$ to $L_{\text {uni }}$ is non-zero. As at the end of the previous section we can write $A=\pi^{*}(\bar{A}) \otimes \mathcal{L}$ where $\bar{A}$ lies in the series of $L_{\text {ad }}$ defined by $\bar{s} \in T^{*} \cap L_{\text {der }}^{*}$ and $\mathcal{L}$ is pulled back from a local system on $L / L_{\text {der }}$. Let $A, \bar{A}$ lie in the series defined by $s, \bar{s} \in T^{*}$, respectively.

It is clear that $A$ and $\pi^{*}(\bar{A})$ have the same restriction to $L_{\text {uni }}$ (cf. [8], (2.6) (c)). Moreover, the restriction of the decomposition in (2.7) to $G_{\text {uni }}$ is related to the restriction of that in (a) by the following formula, see [8], (2.6) (e):

$$
K_{E}^{s}=\bigoplus_{E^{\prime}}\left(E: E^{\prime}\right) K_{E^{\prime}}^{\bar{s}} \quad \text { on } G_{\text {uni }}
$$


where $E \in \operatorname{Irr}\left(W_{L, s}^{G}\right), E^{\prime} \in \operatorname{Irr}\left(W_{L}^{G}\right)$ and $\left(E: E^{\prime}\right)$ denotes the multiplicity of $E$ in the restriction of $E^{\prime}$ to $W_{L, s}^{G}$.

\section{The evaluation of certain class functions.}

From now we assume that $G$ has a fixed $\mathbb{F}_{q}$-rational structure with Frobenius map $F$ and that $T$ is a maximally-split torus, that is, an $F$-stable maximal torus contained in some $F$-stable Borel subgroup $B$ of $G$. Let $S \subset W$ be the set of standard generators determined by $B$. Then $F$ induces an automorphism of $(W, S)$ which will again be denoted by $F$. Since $G$ is defined over $\mathbb{F}_{q}$ then the same holds for $G^{*}$ and we denote again by $F$ the corresponding Frobenius morphism. Moreover, there exists a maximallysplit torus $T^{*}$ contained in some $F$-stable Borel subgroup $B^{*} \subseteq G^{*}$, 'in duality' with the pair $T \subseteq B$ (see [3], Section 4.3). We can identify the pair $(W, S)$ with the corresponding pair $\left(W^{*}, S^{*}\right)$ in $G^{*}$ (defined with respect to $\left.T^{*} \subseteq B^{*}\right)$ such that the action of $F$ on $W$ corresponds to the action of $F^{-1}$ on $W^{*}$. We assume that $G$ has connected center. That fact implies that centralizers of semisimple elements in $G^{*}$ are connected, and these properties are inherited by Levi subgroups of parabolic subgroups of $G$.

A complex $K \in \mathcal{D} G$ is said to be $F$-stable if $F^{*} K \simeq K$. For an $F$-stable complex $K$, with a given isomorphism $\varphi: F^{*} K \stackrel{\sim}{\rightarrow} K$, following [7], (8.4), we define a characteristic function $\chi_{K, \varphi}: G^{F} \rightarrow \overline{\mathbb{Q}}_{\ell}$ by

$$
\chi_{K, \varphi}(x)=\sum_{i}(-1)^{i} \operatorname{Tr}\left(\varphi, \mathcal{H}_{x}^{i}(K)\right)
$$

where $\mathcal{H}_{x}^{i}(K)$ denotes the stalk at $x \in G^{F}$ of $i$-th cohomology sheaf $\mathcal{H}^{i}(K)$ of $K$, and $\varphi$ is the induced linear map on $\mathcal{H}_{x}^{i}(K)$. If $K$ is a $G$-equivariant perverse sheaf, $\chi_{K, \varphi}$ gives rise to a class function on $G^{F}$.

(3.1) We denote by $(\Sigma, \mathcal{E})$ the cuspidal pair for $L$ (in the sense of [6], Definition 2.4) such that the (shift of) intersection cohomology complex $\operatorname{IC}(\bar{\Sigma}, \mathcal{E})[\operatorname{dim} \Sigma]$, extended to the whole of $L$ by zero on $L-\bar{\Sigma}$ (which is a cuspidal character sheaf on $L$ ) belongs to $\hat{L}_{s}$. Following Lusztig [8], Section 3.2, we choose an isomorphism $F^{*} \mathcal{E} \stackrel{\sim}{\rightarrow} \mathcal{E}$ inducing the map $q^{\frac{1}{2}\left(\operatorname{dim}\left(L / \mathrm{Z}(L)^{0}\right)-\operatorname{dim} \Sigma\right)}$ times identity on the stalk over $u$ of $\mathcal{E}$, where $u \in \Sigma\left(\mathbb{F}_{q}\right)$ with $F$ acting trivially on the set of irreducible components of the variety $\mathcal{B}_{u}$ of Borel subgroups containing $u$. The induced complex inherits a natural mixed structure. The canonical induced mixed structure on the cuspidal character sheaf on $L$ is compatible with the isomorphism (2.7). We denote by $\chi_{K_{E}^{s}}$ and $\chi_{K_{E^{\prime}}^{\bar{s}}}$ the corresponding characteristic functions, with the notations of (2.7) and (2.9 (a)). 
Using (2.9 (b)) we obtain

$$
\chi_{K_{E}^{s}}=\sum_{E^{\prime}}\left(E: E^{\prime}\right) \chi_{K_{E^{\prime}}^{s}},
$$

where $E^{\prime} \in \operatorname{Irr}\left(W_{L}^{G}\right)$ and $\left(E: E^{\prime}\right)$ denotes the multiplicity of $E$ in the restriction of $E^{\prime}$ to $W_{L, s}^{G} \subseteq W_{L}^{G}$.

Let $\mathcal{N}_{G}$ be the set of pairs $(C, \mathcal{E})$ where $C$ is a unipotent class of $G$ and $\mathcal{E}$ is an irreducible local system on $C$ which is $G$-equivariant for the conjugation action of $G$. From the list in the introduction of [6] we see that the set $\mathcal{N}_{G}$ contains at most one cuspidal pair, and if such a pair exists then the rank of $G / Z(G)$ is even. We shall denote by $C^{\prime} \leq C$ the usual partial order on unipotent classes. The local system $\mathcal{E}$ has a natural mixed structure.

We define a class function $Y_{C, \mathcal{E}}: G^{F} \rightarrow \overline{\mathbb{Q}}_{\ell}$ as follows:

$$
Y_{C, \mathcal{E}}(g):=\chi_{\mathrm{IC}(\bar{C}, \mathcal{E})}(g), \quad g \in \bar{C}^{F},
$$

extended by zero on $G^{F}-\bar{C}^{F}$ (where the mixed structure on $\operatorname{IC}(\bar{C}, \mathcal{E})$ is that extending the one given by the above distinguished isomorphism $\left.F^{*} \mathcal{E} \stackrel{\sim}{\rightarrow} \mathcal{E}\right)$.

Let $E^{\prime} \in \operatorname{Irr}\left(W_{L}^{G}\right)$. We shall denote by $\left(C_{E^{\prime}}, \mathcal{E}_{E^{\prime}}\right)$ the pair which corresponds with $E^{\prime}$ under the generalized Springer correspondence (see [6], Theorem 6.5 and [1] for the explicit description of that correspondence). We shall set $Y_{E^{\prime}}:=Y_{C_{E^{\prime}}, \mathcal{E}_{E^{\prime}}}$ and we shall denote by $\operatorname{dim} \mathcal{B}_{E^{\prime}}$ the dimension of the variety of Borel subgroups of $G$ containing a fixed element in the class $C_{E^{\prime}}$.

A unipotent element $u$ of $G^{F}$ is said split if $F$ acts trivially on the set of irreducible components of the variety of Borel subgroups containing $u$. The assumption that $p$ is good remains in force. Then it is known $[13,12,2]$ that for each $F$-stable unipotent clas $C$ of $G$ there exists a unique split element $u \in C^{F}$ up to $G^{F}$-conjugacy except in the following case, i.e., $G$ of type $E_{8}$, $q \equiv-1(\bmod 3)$ and $u$ is of type $D_{8}\left(a_{3}\right)$ (notation of [3]). In this exceptional case, split elements do not exist in $C^{F}$. From now we are assuming that $p$ is good and that if $G$ of type $E_{8}, q \not \equiv-1(\bmod 3)$.

Theorem 3.2. Let $s \in T^{*}$ and $E \in \operatorname{Irr}\left(W_{L, s}^{G}\right)$. We assume that the element $s \in T^{*}$ is isolated in $G^{*}$ and that the set $\hat{L}_{s}^{0} \cap \hat{L}_{\mathrm{uni}}^{0}$ is non-empty. Let $C$ be an F-stable unipotent class in $G$ and let $u \in C^{F}$ be a split element. Then

$$
\chi_{K_{E}^{s}}(u)=\sum_{E^{\prime}} m_{E^{\prime}}(-1)^{\operatorname{rank} G} q^{\operatorname{dim} \mathcal{B}_{E^{\prime}}+\frac{1}{2} \operatorname{srank} L} Y_{E^{\prime}}(u),
$$

where $E^{\prime} \in \operatorname{Irr}\left(W_{L}^{G}\right)$, where $m_{E^{\prime}}$ denotes the multiplicity of $E$ in the restriction of $E^{\prime}$ to $W_{L, s}^{G} \subseteq W_{L}^{G}$ and srank $L$ denotes the semisimple rank of $L$. 
Proof. By $(2.9$ (b)), it is sufficient to check that

$$
\chi_{K_{E^{\prime}}^{\bar{s}}}(u)=(-1)^{\operatorname{rank} G} q^{\operatorname{dim} \mathcal{B}_{E^{\prime}}+\frac{1}{2} \operatorname{srank} L} Y_{E^{\prime}}(u),
$$

for any $E^{\prime} \in \operatorname{Irr}\left(W_{L}^{G}\right)$.

First note that the center $\mathrm{Z}(L)$ of $L$ is connected (since the center of $G$ is), that $L / \mathrm{Z}(L)$ is simple and the rank of $L / Z(L)$ is even (since the set $\hat{L}_{s}^{0} \cap \hat{L}_{\text {uni }}^{0}$ is non-empty).

Then, according to [6], Theorem 6.5,

$$
K_{\left.E^{\prime}\right|_{G_{\mathrm{uni}}}}^{\bar{s}} \simeq \mathrm{IC}\left(\bar{C}_{E^{\prime}}, \mathcal{E}_{E^{\prime}}\right)\left[\operatorname{dim} C_{E^{\prime}}+\operatorname{dim} \mathrm{Z}(L)\right]
$$

extended by zero on $G_{\mathrm{uni}}-\bar{C}_{E^{\prime}}$. The restriction of $\mathcal{H}^{\operatorname{dim} C_{E^{\prime}}-\operatorname{dim} \mathrm{Z}(L)}\left(K_{E^{\prime}}^{\bar{s}}\right)$ to $C_{E^{\prime}}$ is isomorphic to $\mathcal{E}_{E^{\prime}}$, the canonical mixed structure on $K_{E^{\prime}}^{\bar{s}}$ induces by this way a mixed structure on $\mathcal{E}_{E^{\prime}}$ and Lusztig has shown in [8], (3.4) (a) that this mixed structure has the property that $F^{*} \mathcal{E}_{E^{\prime}} \stackrel{\sim}{\rightarrow} \mathcal{E}_{E^{\prime}}$ induces on the stalk over a split element of $C_{E^{\prime}}$ the identity map times $q^{\frac{m}{2}}$ where

$$
m=\operatorname{dim} G-\operatorname{dim} C_{E^{\prime}}-\operatorname{dim} \mathrm{Z}(L) .
$$

Since $\operatorname{dim} \mathrm{Z}(L)=\operatorname{rank} L-\operatorname{srank} L \operatorname{rank} L=\operatorname{rank} G$, we get

$$
\operatorname{dim} C_{E^{\prime}}+\operatorname{dim} \mathrm{Z}(L)=\operatorname{dim} C_{E^{\prime}}+\operatorname{rank} G-\operatorname{srank} L .
$$

Hence, using that $\operatorname{dim} C_{E^{\prime}}$ and $\operatorname{rank} L / Z(L)$ are even, we see the sign corresponding with the shift is $(-1)^{\operatorname{rank} G}$.

Next the following formula (see [16], page 54)

$$
2 \operatorname{dim} \mathcal{B}_{E^{\prime}}=\operatorname{dim} C_{E^{\prime}}+\operatorname{rank} G-\operatorname{dim} G,
$$

joint to the above value for $m$, provides us the power of $q$.

Remark 3.3. Let us state the following property.

(P) There exists an irreductible representation $E_{1}^{\prime}$ of $W_{L}^{G}$ such the following hold.

(a) The representation $E_{1}^{\prime}$ occurs with multiplicity one in the induced representation $\operatorname{Ind}_{W_{L, s}^{G}}^{W_{L}^{G}}(E)$;

(b) If $E^{\prime}$ occurs in $\operatorname{Ind}_{W_{L, s}^{G}}^{W_{G}^{G}}(E)$, then $\operatorname{dim} C_{E^{\prime}} \leq \operatorname{dim} C_{E_{1}^{\prime}}$, with equality only for $E^{\prime}=E_{1}^{\prime}$. 
In [8], (4.10), Lusztig sketched the proof of Property (P) for groups of types $B_{n}$. In [1] we give a complete proof of that fact and we also prove that Property (P) holds for other groups of classical type (for type $C_{n}$, we need to assume that $t \neq 0$, i.e., that $L \neq T$ ). Note that for exceptional groups (see 2.6) the only case to consider is $L=T$.

When Property (P) holds, Theorem 3.2 implies that

$$
\begin{aligned}
\chi_{K_{E}^{s}}(u)=(-1)^{\operatorname{rank} G} q^{\operatorname{dim} \mathcal{B}_{E^{\prime}}+\frac{1}{2} \operatorname{srank} L} & \\
& + \text { some linear combination of } Y_{\left(C^{\prime}, \mathcal{E}\right)}(u),
\end{aligned}
$$

where $C^{\prime}$ are unipotent classes of $G$ such that $\operatorname{dim} C^{\prime} \leq \operatorname{dim} C_{E_{1}^{\prime}}$.

In that case, we are able to define a map

$$
\text { Sup : } \hat{G}_{\text {uni }} \longrightarrow\{\text { unipotent classes of } G\}
$$

by composition of the inverse of the map defined in (2.8) and of the map

$$
(L, s, E) \mapsto C_{E_{1}^{\prime}}
$$

(3.4) By $[7,(17.8 .3)]$ we have a bijection

$$
\hat{G}_{s} \longleftrightarrow \coprod_{\mathcal{F}} \mathcal{M}\left(\mathcal{G}_{\mathcal{F}}\right), \quad \text { together with injections } \quad \mathcal{F} \hookrightarrow \mathcal{M}\left(\mathcal{G}_{\mathcal{F}}\right),
$$

where $\mathcal{F}$ runs over the families of irreducible characters of $W_{s}, \mathcal{G}_{\mathcal{F}}$ is a finite group, and $\mathcal{M}\left(\mathcal{G}_{\mathcal{F}}\right)$ is the set of $\mathcal{G}_{\mathcal{F}}$-conjugacy classes of pairs $(x, \sigma)$ where $x \in \mathcal{G}_{\mathcal{F}}$ and $\sigma$ is an irreducible representation of the centralizer of $x$ in $\mathcal{G}_{\mathcal{F}}$. (For the definition of families, see [5], Chap. 4.)

(3.5) Define a function $\mathcal{M}\left(\mathcal{G}_{\mathcal{F}}\right) \rightarrow \overline{\mathbb{Q}}_{\ell}{ }^{\times}$by $m:=(x, \sigma) \mapsto \lambda_{m}$, where $m$ is the scalar by which $x$ acts on the representation $\sigma$ of the centralizer of $x$ in $\mathcal{G}_{\mathcal{F}}$ (see [5], 11.1). Then $\lambda_{m}$ is a root of 1 dor any $m \in \mathcal{M}\left(\mathcal{G}_{\mathcal{F}}\right)$. We assume that $\mathcal{G}_{\mathcal{F}}$ is a vector space of dimension $d$ over $\mathbb{F}_{2}$ (it is always the case if $G$ is of classical type). Then $\mathcal{M}\left(\mathcal{G}_{\mathcal{F}}\right)$ is a vector space of dimension $2 d$ over $\mathbb{F}_{2}$ and there is a unique quadratic form $Q$ on it, with values in $\mathbb{F}_{2}$, such that $\lambda_{m}=(-1)^{Q(m)}$ for any $m \in \mathcal{M}\left(\mathcal{G}_{\mathcal{F}}\right)$ and such that

$$
\left\{m, m^{\prime}\right\}=2^{-d}(-1)^{Q\left(m+m^{\prime}\right)+Q(m)+Q\left(m^{\prime}\right)},
$$

where $\{\}:, \mathcal{M}\left(\mathcal{G}_{\mathcal{F}}\right) \times \mathcal{M}\left(\mathcal{G}_{\mathcal{F}}\right) \rightarrow \overline{\mathbb{Q}}_{\ell}$ is the pairing defined by Lusztig in [5], (4.14.3). 
We now state another property in order to describe a way to get the whole of [8], (1.6).

(L) Let $m_{E}$ be the element of $\mathcal{M}\left(\mathcal{G}_{\mathcal{F}}\right)$ which corresponds with $K_{E}^{s}$ under the bijection (3.4). Then there is a Lagrangian subspace $X_{\mathcal{M}\left(\mathcal{G}_{\mathcal{F}}\right)}$ of $\mathcal{M}\left(\mathcal{G}_{\mathcal{F}}\right)$ and a unipotent class $C$ of $G$ which is canonically associated with $X_{\mathcal{M}\left(\mathcal{G}_{\mathcal{F}}\right)}$ such that the following hold.

(a) If $E^{\prime}$ occurs in the induced representation $\operatorname{Ind}_{W_{L, s}^{G}}^{W_{L}^{G}}(E)$ and if $m_{E} \notin$ $X_{\mathcal{M}\left(\mathcal{G}_{\mathcal{F}}\right)}$ then $\bar{C}_{E^{\prime}} \not \supset C$;

(b) If $m_{E} \in X_{\mathcal{M}\left(\mathcal{G}_{\mathcal{F}}\right)}$, there exists a unique irreductible representation $E_{1}^{\prime}$ of $W_{L}^{G}$ occuring in $\operatorname{Ind}_{W_{L, s}^{G}}^{W_{G}^{G}}(E)$ such that $\bar{C}_{E_{1}^{\prime}} \supset C$; in this case it occurs with multiplicity one and $C_{E_{1}^{\prime}}=C$.

Note that, in case $\mathcal{F}$ is a cuspidal family (see [5], (8.1)), it is in some sense the counterpart of [15], (6.12.1).

Corollary 3.6. Assume that property (L) holds. Let $u \in C^{F}$ be a split element. Then, under the assumptions of Theorem 3.2,

$$
\chi_{K_{E}^{s}}(u)= \begin{cases}(-1)^{\operatorname{rank} G} q^{\operatorname{dim} \mathcal{B}_{E_{1}^{\prime}}+\frac{1}{2} \operatorname{srank} L} & \text { if } m_{E} \in X_{\mathcal{M}\left(\mathcal{G}_{\mathcal{F}}\right)}, \\ 0 & \text { if } m_{E} \notin X_{\mathcal{M}\left(\mathcal{G}_{\mathcal{F}}\right)} .\end{cases}
$$

Proof. It follows clearly from Theorem 3.2.

Remark 3.7. In [15, Lemma 6.11], Shoji proved similar results for unipotent character sheaves (that is the case $s=1$ ) without condition on support.

\section{Dual characterization.}

In Remark 3.3 (under the assumption that Property (P) holds true) we have defined a map Sup from the set of isomorphism classes of characters sheaves $A \in \hat{G}$ such that the restriction of $A$ to $G_{\text {uni }}$ is non-zero to the set of unipotent classes. In [7], Theorem 10.7, Lusztig defined a map from all the set $\hat{G}$ (i.e., without assuming non-zero restriction to the unipotent variety) to the set of unipotent classes.

Let us summarize Lusztig's construction. By [7], Corollary 16.7, there exists a well-defined map

$$
\hat{G}_{s} \longrightarrow\left\{\text { two-sided cells in } W_{s}\right\} .
$$

(For the definition of two-sided cells, see [5], pages 137 and 160. For the link of that map with the bijection (3.4), see [5], Theorem 5.25.) 
The subset of $\hat{G}_{s}$ consisting of all character sheaves which belong to the two-sided cell $\underline{c}$ of $W_{s}$ will be denoted by $\hat{G}_{s, \underline{c}}$. (Note that $\hat{G}_{s, \underline{c}}$ is a finite set which is a symplectic vector space over $\mathbb{F}_{2}$ or a non abelian analogue.) Then Lusztig [11], (10.5), describes a canonical construction by which we can associate with a pair $(s, \underline{c})$ (where $s \in T^{*}$ and $\underline{c}$ is a two-sided cell in $W_{s}$ ) a well-defined unipotent class $C$ in $G$. This is done as follows. (We assume here the center of $G$ is connected, for the general case see [11], (10.4).)

Let $Y$ be the group of one parameter subgroups of $T$. Then $Y$ is naturally a module for $W$. By restriction $Y$ is also a module for $W_{s}$. There is a welldefined irreducible representation of $W_{s}$ which corresponds to $\underline{c}$. (We denote it as $E(\underline{c})$ and we call it the special representation of $W_{s}$ corresponding to $\underline{c}$.) Let $b_{E(\underline{c})}$ be the smallest non-negative integer $i$ such that the $W_{s^{-}}$ module $E(\underline{c})$ occurs in the $i$-th symmetric power of $Y$. (Then $E(\underline{c})$ occurs with multiplicity one in the $b_{E(\underline{c})}$-th symmetric power of $Y$.) There exists a unique irreducible representation $E$ of $W$ such that $E$ occurs with non-zero multiplicity both in the representation induced from $E(\underline{c})$ and in the $b_{E(\underline{c})^{-}}$th symmetric power of the $W$-module $Y$.

Under Springer's correspondence the representation $E$ corresponds to a pair consisting of a unipotent class $C$ in $G$ and an irreducible, $G$-equivariant local system $\mathcal{E}$ on $C$. Thus, we can define a map

$$
\begin{aligned}
\Phi_{G}:\left\{(s, \underline{c}) \mid s \in T^{*} \text { and } \underline{c} \text { two-sided cell in } W_{s}\right\} & \\
& \longrightarrow\{\text { unipotent classes in } G\}
\end{aligned}
$$

by associating with the pair $(s, \underline{c})$ the unipotent class $C$.

Lusztig proved the following result (without assumption of connected center) for $p$ "large enough" (that is sufficiently large, so that we can operate with the Lie algebra of $G$ as if we were in characteristic zero).

Theorem 4.1 (Cf. [11], Theorem 10.7 and [9], (4.6)). Let $s \in T^{*}$ and let $\underline{c}$ be a two-sided cell in $W_{s}$. Let $C=\Phi_{G}(s, \underline{c})$. Then the following hold.

(a) If $A \in \hat{G}_{s, \underline{c}}$ and $C^{\prime}$ is a unipotent class such that $A_{\left.\right|_{\{g\}}} \neq 0$ for some $g \in C^{\prime}$ then $\operatorname{dim} C^{\prime} \leq \operatorname{dim} C$, with equality only for $C^{\prime}=C$.

(b) There exists some $A \in \hat{G}_{s, \underline{c}}$ and some $g \in C$ such that $A_{\left.\right|_{\{g\}}} \neq 0$.

Assuming that Property $(\mathrm{P})$ holds true and that $p$ is large enough to apply the results of [11], by the same argument as [11], proof of Theorem 10.7 (i), page 173 , we obtain

$$
\operatorname{Sup}(A)=\Phi_{G}(s, \underline{c}) .
$$

It is natural to guess more generally that the equality $(*)$ holds true in good characteristic (with the assumption $q \not \equiv-1(\bmod 3)$ in case $G$ of type $\left.E_{8}\right)$. 
Note that when $(*)$ holds true, it gives a link between the two classifications of character sheaves with non-zero restriction to the unipotent variety.

\section{Appendices.}

5.1. Appendix A. Cuspidal character sheaves. The following properties have been used in the proof of Fact 2.3. We have extracted them from [7] for helping the reader in reading our paper.

We assume now that $s$ is a given semisimple element of $G^{*}$ such that the set $\hat{G}_{s}$ contains a cuspidal character sheaf (denoted by $A$ ). Then the following hold:

(a) The semisimple element $s$ is isolated.

(b) The family which corresponds to $A$ is cuspidal in the sense of [7], (23.15) (see [7], (17.13.11)).

Type $A_{n}$. In this case $G$ does not have any cuspidal character sheaf at all unless $n=0$.

Type $B_{n}$. We assume that $G=\mathrm{SO}_{2 n+1}(k)$. Then $G^{*}=\operatorname{Sp}_{2 n}(k)$. Since $p$ is odd and $G$ has a cuspidal character sheaf, the group $W_{s}$ is of type $C_{r} \times C_{r^{\prime}}$, where $r+r^{\prime}=n$, with $\left(r \geq 1\right.$ and $r^{\prime} \geq 1$ ) (see [7], 23.5. (i)) and $G$ has a cuspidal family. From [7], 23.16 (i), it follows that the number $r$ (resp. $r^{\prime}$ ) is twice a triangular number. Each series $\hat{G}_{s}$ contains at most one cuspidal character sheaf and, for each pair $\left(r, r^{\prime}\right)$ as above there is a (unique) series $\hat{G}_{s}$ such that the group $W_{s}$ is of type $C_{r} \times C_{r^{\prime}}$ which contains a cuspidal character sheaf (see [7], 23.17 and $23.19\left(c_{1}\right)$ ). The cuspidal character sheaves on $G$ are supported by the closure of the conjugacy class of $\sigma v$, where $\sigma$ is a semisimple element of $G$ with $\mathrm{C}_{G}^{0}(\sigma)$ isomorphic to, or doubly covered by $\mathrm{SO}_{\left(d+d^{\prime}+1\right)^{2}}(k) \times \mathrm{SO}_{\left(d-d^{\prime}\right)^{2}}(k)$, with $r=d^{2}+d$ and with $r^{\prime}=d^{\prime 2}+d^{\prime}$ and $v$ is a certain unipotent element in $\mathrm{C}_{G}^{0}(\sigma)$; by using [7], 23.2 (c) and 23.19 $\left(c_{1}\right)$, we see that $\sigma=1$ if and only if $r=r^{\prime}$ (note that in this case, setting $r=r^{\prime}=t^{2}+t$, we get $\left.2 n+1=4 t^{2}+4 t+1=(2 t+1)^{2}\right)$. Each $G$-conjugacy class $(\sigma v)_{G}$ as above is $F$-stable.

Type $C_{n}$. We assume that $G=\operatorname{PSp}_{2 n}(k)$ (with $n \geq 1$ ). Then $G^{*}=$ $\operatorname{Spin}_{2 n+1}(k)$. Since $p$ is odd and $G$ has a cuspidal character sheaf, the integer $n$ is even (see [7], 23.2.(a)) and the group $W_{s}$ is of type $D_{r} \times B_{r^{\prime}}$, where $r+r^{\prime}=n$, with $\left(r \geq 2\right.$ and $\left.r^{\prime} \geq 1\right)$ or $r=0$ or $r^{\prime}=0$ (see [7], 23.3. (i) and (ii)) and has a cuspidal family. By [7], 23.16 (i) and (ii), it follows that $r^{\prime}$ is twice a triangular number and that $r$ is an even (since $n$ is even) square. Each series $\hat{G}_{s}$ contains at most one cuspidal character sheaf. For each pair $\left(r, r^{\prime}\right)$ as above with $r \neq 0$, there is a (unique) series $\hat{G}_{s}$ such that $W_{s}$ is of type $D_{r} \times C_{r^{\prime}}$ which contains a cuspidal character sheaf. There are two series $\hat{G}_{s}$ and $\hat{G}_{s^{\prime}}$ such that $W_{s}=W_{s^{\prime}}=W$ (see [7], 23.17 and 23.19 
(a)). The cuspidal character sheaves on $G$ are supported by the closure of the conjugacy class of $\sigma u$ where $\sigma$ is a semisimple element of $G$ with $\mathrm{C}_{G}^{0}(\sigma)$ doubly covered by $\operatorname{Sp}_{\left(d+d^{\prime}\right)\left(d+d^{\prime}+1\right)}(k) \times \operatorname{Sp}_{\left(d-d^{\prime}-1\right)\left(d-d^{\prime}\right)}(k)$, with $r=d^{2}$ and $r^{\prime}=d^{\prime 2}+d^{\prime}$, and $v$ is a certain unipotent element of $\mathrm{C}_{G}^{0}(\sigma)$; setting $r=a^{2}$ and $r^{\prime}=a^{\prime 2}+a^{\prime}$, by using [7], 23.2(a) and (23.19.1), we see that $\sigma=1$ if and only if $a=a^{\prime}$ or $a=a^{\prime}+1$ (note that in this case, setting $a=2 t$ ( $a$ is always even since $r$ is), we get $2 n=16 t^{2}+4 t$ or $\left.2 n=16 t^{2}-4 t\right)$. Each $G$-conjugacy class $(\sigma v)_{G}$ as above is $F$-stable. In the case $r=0$, the cuspidal character sheaf which belongs to $\hat{G}_{s^{\prime}}$ is equal to the tensor product by a tame local system of rank one of the one which belongs to $\hat{G}_{s}$ (they have the same support) and at least one of the two is $F$-stable.

Type $D_{n}$. We assume that $G=\operatorname{PSO}_{2 n}(k)$. Then $G^{*}=\operatorname{Spin}_{2 n}(k)$. Since $p$ is odd and $G$ has a cuspidal character sheaf, the integer $n$ is divisible by 4 (see [7], 23.2.(c)), the group $W_{s}$ is of type $D_{r} \times D_{r^{\prime}}$, where $r+r^{\prime}=n$, with $(r \geq 2$ and $r^{\prime} \geq 2$ ) or $r=0$ or $r^{\prime}=0$ (see [7], 23.5. (ii)) and has a cuspidal family. It follows from [7], 23.16 (ii) that $r$ and $r^{\prime}$ are even (since $n$ is divisible by 4) squares. Each series $\hat{G}_{s}$ contains at most one cuspidal character sheaf and, for each pair of integers $\left(r, r^{\prime}\right)$ such that $r \neq r^{\prime}$ and $r r^{\prime} \neq 0$, there is a (unique) series $\hat{G}_{s}$ with $W_{s}$ of type $D_{r} \times D_{r^{\prime}}$ which contains a cuspidal character sheaf. There are two series $\hat{G}_{s}$ and $\hat{G}_{s^{\prime}}$ such that $r=r^{\prime}$ and there are four series $\hat{G}_{s}$, $\hat{G}_{s^{\prime}}, \hat{G}_{s^{\prime \prime}}$ and $\hat{G}_{s^{\prime \prime \prime}}$ such that $W_{s}=W_{s^{\prime}}=W_{s^{\prime \prime}}=W_{s^{\prime \prime \prime}}=W$ (see [7], 23.17 and $\left.23.19\left(c_{2}\right)\right)$. The cuspidal characters sheaves on $G$ are supported by the closure of the conjugacy class of $\sigma v$ where $\sigma$ is a semisimple element of $G$ with $\mathrm{C}_{G}^{0}(\sigma)$ isomorphic to, or doubly covered by, $\mathrm{SO}_{\left(d+d^{\prime}\right)^{2}}(k) \times \mathrm{SO}_{\left(d-d^{\prime}\right)^{2}}(k)$, with $r=d^{2}$ and $r^{\prime}=d^{\prime 2}$, and $v$ is a certain unipotent element of $\mathrm{C}_{G}^{0}(\sigma)$; by using [7], 23.2(c) and (23.19.2), we see that $\sigma=1$ if and only if $r=r^{\prime}$ (note that in this case, setting $r=r^{\prime}=(2 t)^{2}$, we get $2 n=16 t^{2}$ ). Each $G$-conjugacy class $(\sigma v)_{G}$ as above is $F$-stable, there is an $F$-stable cuspidal character sheaf $A$ of $G$ which is supported by the closure of it and the other cuspidal characters sheaves which are supported by the closure of the same conjugacy class (when they exist) are obtained by tensoring $A$ by tame local systems on $G$ of rank one corresponding to central elements of $G^{*}$.

Type $G_{2}$. Assume that $G$ is simple of type $G_{2}$. The group $G$ has four cuspidal characters sheaves. All of them are unipotent (see the proof of [7], Proposition 20.6) and $F$-stable. One of them has non-zero restriction to $G_{\text {uni }}$ (it is is supported by the closure of the subregular unipotent class in $G$ ).

Type $F_{4}$. Assume that $G$ is an adjoint group of type $F_{4}$. The group $G$ has seven cuspidal character sheaves (see [7], proof of Proposition 21.3). All of them are unipotent and $F$-stable. One of them has non-zero restriction to $G_{\text {uni }}$. 
Type $E_{6}$. Assume that $G$ is an adjoint group of type $E_{6}$. The group $G$ has six cuspidal character sheaves: There are three series corresponding to central semisimple elements of $G^{*}$ and each such series contains two cuspidal character sheaves (see [7], proof of Proposition 20.3 (a)). Thus if $\hat{G}_{s}$ contains a cuspidal character sheaf, then $s$ must be central. The six cuspidal character sheaves have the same support: The closure of the conjugacy class of $\sigma v$ where $\sigma$ is a $F$-stable semisimple element of $G$ whose connected centralizer $\mathrm{C}_{G}^{0}(\sigma)$ is isogenous to $\mathrm{SL}_{3}(k) \times \mathrm{SL}_{3}(k) \times \mathrm{SL}_{3}(k)$ and $v$ is a regular $F$-stable unipotent element in $\mathrm{C}_{G}^{0}(\sigma)$.

Type $E_{7}$. Assume that $G$ is an adjoint group of type $E_{7}$. The group $G$ has four cuspidal character sheaves: There are two series corresponding to central semisimple elements of $G^{*}$ and each such series contains two cuspidal character sheaves (see [7], proof of Proposition 20.3 (c)). Thus if $\hat{G}_{s}$ contains a cuspidal character sheaf, then $s$ must be central. The four cuspidal character sheaves have the same support: The closure of the conjugacy class of $\sigma v$ where $\sigma$ is a semisimple element of $G$ whose connected centralizer $\mathrm{C}_{G}^{0}(\sigma)$ is isogenous to $\mathrm{SL}_{4}(k) \times \mathrm{SL}_{4}(k) \times \mathrm{SL}_{2}(k)$ and $v$ is a regular unipotent element in $\mathrm{C}_{G}^{0}(\sigma)$.

Type $E_{8}$. Assume that $G$ is a simple group of type $E_{8}$. It has 13 cuspidal character sheaves (see [6], Section 15.6). All of them are unipotent (see [9], 4.7. (a)) and F-stable. One of them is supported by the closure of a unipotent class.

5.2. Appendix B. On Jordan decompositions for character sheaves. The aim of this appendix is an application of (2.5). Let $s \in T^{*}$. Let $G(s)$ be a group dual to $\mathrm{C}_{G^{*}}(s)$ Let $L$ be a standard Levi subgroup of $G$ and let $L(s)$ be the corresponding standard Levi subgroup of $G(s)$, dual to $\mathrm{C}_{L^{*}}(s)$. Let $s \in T^{*}$ such that $\hat{L}_{s}^{0}$ is not empty, and let $A \in \hat{L}_{s}^{0}$. We denote by $\hat{G}_{L, s}$ the set of character sheaves which are components of the induced complex $\operatorname{ind}_{L}^{G}(A)$. Then it follows from (2.5) that there exist bijections

$$
I_{L, s}^{G}: \operatorname{Irr}\left(W_{L, s}^{G}\right) \rightarrow \hat{G}_{L, s}, \quad I_{L(s), 1}^{G(s)}: \operatorname{Irr}\left(W_{L(s), 1}^{G(s)}\right) \rightarrow \widehat{G(s)}_{L(s), 1} .
$$

Proposition 5.1 [Jordan decomposition]. The following hold.

(a) The sets $\hat{G}_{s}^{0}$ and $\left.\widehat{G(s)}\right)_{1}^{0}$ have the same cardinality.

(b) Any bijection $J_{L, s}^{L}: \hat{L}_{s}^{0} \rightarrow \widehat{L(s)}{ }_{1}^{0}$ can be extended to a bijection

$$
J_{L, s}^{G}: \hat{G}_{L, s} \rightarrow \widehat{G(s)} L(s), 1
$$

by setting

$$
J_{L, s}^{G}=I_{L(s), 1}^{G(s)} \circ\left(I_{L, s}^{G}\right)^{-1}
$$


(c) Let $J_{L, s}^{G}$ be a bijection as in (b) and let $M$ be any standard Levi subgroup of $G$ which contains $L$. We have

$$
J_{L, s}^{G} \circ \operatorname{Ind}_{M}^{G}=\operatorname{Ind}_{M(s)}^{G(s)} \circ J_{L, s}^{M} .
$$

(d) Assume that there exists $M \neq G$ as in (c) which contains $\mathrm{C}_{G^{*}}(s)$. Then $\operatorname{Ind}_{M}^{G}$ induces a bijection between $\hat{M}_{s, L}$ and $\hat{G}_{s, L}$.

Proof. The assertion (a) could certainly be extracted from [7], but we can also prove it by an easy induction on the semisimple rank of $G$ by using (b). Now (b) follows from [7], (10.4.5), by working with characteristic functions of character sheaves instead of character sheaves themselves (as in the proof of [7], Proposition 15.2), using the fact (recalled above) that the groups $W_{L, s}^{G}$ and $W_{L(s), 1}^{G(s)}$ can be canonically identified. Next (c) follows from (b) by a standard argument. Finally, it follows from (c), that if $M \supset \mathrm{C}_{G^{*}}(s)$, we have $\operatorname{Ind}_{M}^{G}=\left(J_{L, s}^{G}\right)^{-1} \circ J_{L, s}^{M}$.

Remark 5.2. It is natural to guess that there exists a bijection $J_{L, s}^{G}$ as above which behaves well with the corresponding Fourier pairings associated to $\hat{G}_{s}$ and to $\widehat{G(s)}$, see [7], (17.8.3). We hope to have the opportunity to settle this question elsewhere.

\section{References}

[1] A.-M. Aubert, Character sheaves and generalized Springer correspondence for groups of classical type, preprint, 1997.

[2] W.M. Beynon and N. Spaltenstein, Green functions of finite Chevalley groups of type $E_{n}(n=6,7,8)$, J. of Algebra, 88 (1984), 584-614.

[3] R.W. Carter, Finite groups of Lie type: Conjugacy classes and complex characters, Wiley, New York, 1985.

[4] G. Lusztig, Unipotent characters of the symplectic and odd orthogonal groups over a finite field, Invent. Math., 64 (1981), 263-296.

[5] Characters of reductive groups over a finite field, Annals Math. Studies, 107, Princeton University Press, 1984.

[6] Intersection cohomology complexes on a reductive group, Invent. Math., 75 (1984), 205-272.

[7] Character sheaves, Advances in Math., 56 (1985), 193-237, 57 (1985), 226-265, 57 (1985), 266-315, 59 (1986), 1-63, 61 (1986), 103-155.

[8] - On the character values of finite Chevalley groups at unipotents elements, Journal of Algebra, 104 (1986), 146-194.

[9] Introduction to character sheaves, Proceedings of Symposia in Pure Math., 47 (1987), 165-179. 
[10] Green functions and character sheaves, Annals of Math., 131 (1990), 355408.

[11] $\quad$ A unipotent support for irreducible representations, Advances in Math., 94 (1992), 139-179.

[12] T. Shoji, On the Green polynomials of Chevalley groups of type $F_{4}$, Comm. Algebra, 10 (1982), 505-543.

[13] - On the Green polynomials of classical groups, Invent. Math., 74 (1983), 237-267.

[14] Character sheaves and almost characters of reductive groups, Advances in Math., 111(2) (1995), 244-313; II, 111(2) (1995), 314-354.

[15] Unipotent characters of finite classical groups, in 'Finite Reductive Groups, Related Structures and Representations', (Luminy 1994), M. Cabanes ed., to appear.

[16] N. Spaltenstein, Classes unipotentes et sous-groupes de Borel, Lecture Notes in Math., 946 (1982), Springer, Berlin-Heidelberg-New York.

D.M.I.-École Normale SupÉrieure

U.R.A. 762 DU C.N.R.S.

45 RUE D'Ulm, F-75005 PARIS

E-mail address: aubert@dmi.ens.fr 